\title{
Early Warning Pesticide Monitoring in Nevada's Surface Waters
}

\section{Introduction}

A pesticide is a substance, or mixture of substances, used to kill or control insects, weeds, plant diseases, and other pest organisms (Nevada Department of Agriculture, 2019). Commercial pesticide applicators, farmers, and homeowners apply about 1.1 billion pounds of pesticides annually to agricultural land, non-crop land, and urban areas throughout the United States (Atwood and Paisley-Jones, 2017). Although intended for beneficial uses, there are also risks associated with pesticide applications, including contamination of groundwater and surface-water resources, which can adversely affect aquatic life and water supplies. Pesticides can contaminate groundwater and surface water directly through point sources (spills, disposal sites, or pesticide drift during an application). The main avenue of contamination, however, is indirect by non-point sources, which include agricultural and urban runoff, erosion, leaching from application sites, and precipitation that has become contaminated by upwind applications (fig. 1, Thodal and others, 2009).

\section{Nevada Pesticide Monitoring and Early Warning Program}

To reduce exposure to pesticide compounds, the U.S. Environmental Protection Agency (EPA), through the Federal Insecticide, Fungicide, and Rodenticide Act (FIFRA), requires that all states establish a pesticide management program. The Nevada Department of Agriculture (NDA), with assistance from the EPA, has developed a program of education (Hefner and Donaldson, 2006), regulation (http://agri.nv.gov/ Resources/Regulations/Pest/), and monitoring (Thodal and others, 2009) in the State of Nevada. In a cooperative effort, the NDA and the U.S. Geological Survey (USGS) began drilling boreholes and sampling water from a network of shallow wells in 1997 to characterize pesticide concentrations in groundwater (Thodal and others, 2009). In 2007, the NDA and USGS began collecting samples from a surface-water network. This fact sheet describes the pesticide monitoring of Nevada's surface waters.

There are several pesticides of particular concern to the NDA. This is based on widespread use and chemical characteristics that make these pesticides, or their degradation products, vulnerable to leaching into groundwater and surface-water resources. Once transported into water, there is significant potential for pesticide concentrations to approach or exceed human health or environmental reference levels. The NDA monitors pesticides of concern in coordination with the EPA to further understand annual pesticide usage, pesticide incidents, and potential contamination of water resources. A pesticide is added to NDA's list of pesticides of concern according to the

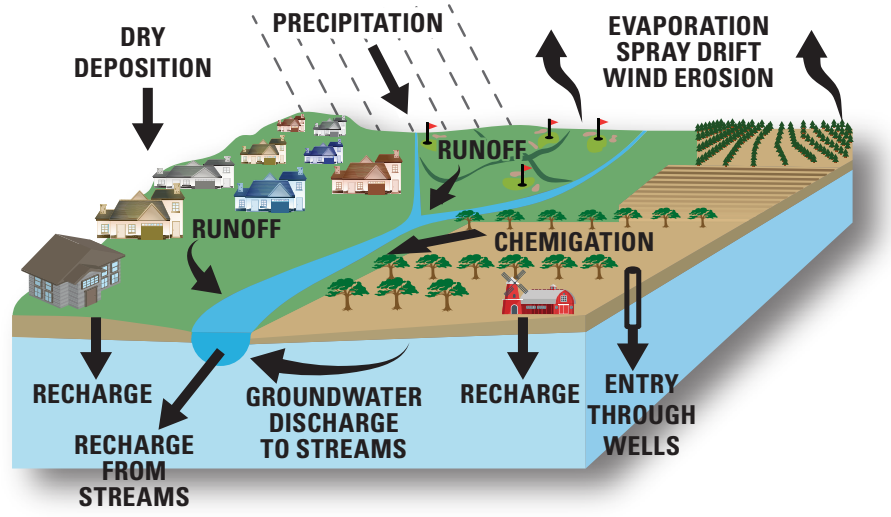

Figure 1. Routes pesticides can travel from the land surface to surface water and groundwater. Chemigation is a process by which pesticides are applied to the land through irrigation systems.

following criteria: (1) frequency of detection, (2) upward trend in the frequency of detection, (3) widespread detections (across multiple counties), and (4) if the highest concentration is above 10 percent of its health and environmental threshold levels (U.S. Environmental Protection Agency, 2018). Pesticides on the list are reevaluated every 5 years and can be removed from the list with continued decreases in the number and severity of detections.

The NDA has developed a pesticide early warning system and best management practices (BMP) tool. The low-level pesticide concentrations collected from surface waters during this study are characterized and synthesized with associated information regarding crop types and land use. Using this as a tool, the NDA educates pesticide applicators, farmers, and the public about how to prevent or reduce pesticide loading early, when the concentrations are low, thereby protecting groundwater and surface-water resources.

\section{Passive Monitoring of Pesticides in Nevada Surface Waters}

Passive methodologies are used to detect low level organic compound concentrations by accumulating and integrating them over time. Passive sampling devices, such as the polar organic chemical integrative sampler (POCIS), trap polar-organic compounds, including some pesticides and their degradates, from the water. As water flows through the POCIS, compounds are trapped and accumulate through time until concentrations are high enough to be detected using standard laboratory analyses (fig. 2; https://www.est-lab.com/pocis.php). The POCIS provides a measure of the absence, presence, or relative abundance of pesticides in the vicinity of the deployed passive sampler (Alvarez, 2010). 
From 2012 to 2019, the NDA and USGS monitored pesticides in Nevada surface waters using POCIS at 26 sites, in 5 hydrographic basins, (table 1; fig. 3). Each sampling event typically consisted of up to six sites in the same watershed. Sites were chosen to be downgradient from agricultural and urban areas and adjacent to existing USGS streamflow gages, which were used to determine streamflow changes during the time POCIS were deployed. POCIS were deployed and retrieved after about 30 days according to the field methods described by

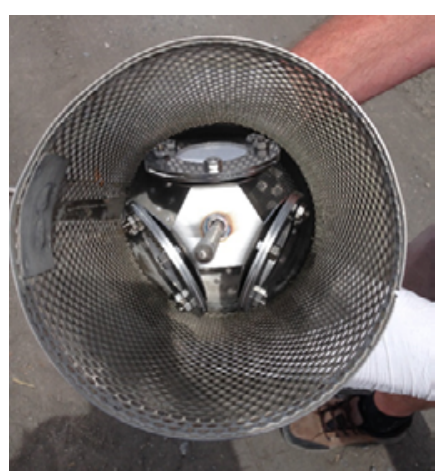

Figure 2. Deployment canister containing three polar organic integrative samplers mounted on a center bracket.
Alvarez (2010). With multiple sampling sites on the same river, relative concentration is used to document pesticide presence. It is assumed that pesticide detections are from possible sources in the upstream vicinity of deployed passive samplers. Pesticide concentrations are compared to available drinking-water criteria and human-health advisories (U.S. Environmental Protection Agency, 2018).

\section{Discrete Monitoring of Pesticides in Nevada Streams}

Discrete samples (collected at a single point in time) of surface waters do not adequately reflect presence or absence of pesticides because pesticide concentrations are typically below analytical reporting levels because of the temporal and spatial variability in pesticide use, runoff, and streamflow (Keith, 1991; Alvarez, 2010). The discrete data provide pesticide concentrations for a "snapshot in time" as opposed to an accumulation of pesticides through a longer period. Although not the preferred collection method for monitoring low level pesticides in this project, discrete samples were collected from the Virgin and Muddy Rivers and the Las Vegas Wash in 2017 because the NDA laboratory was unable to analyze POCIS samples because of mechanical issues with analytical equipment. The discrete samples were analyzed at the USGS National Water Quality Laboratory (NWQL) in Lakewood, Colorado for 225 pesticide compounds (table 1).

Table 1. Areas of pesticide monitoring in Nevada.

\begin{tabular}{|c|c|c|c|c|}
\hline $\begin{array}{l}\text { Surface-water } \\
\text { feature }\end{array}$ & $\begin{array}{l}\text { Hydrographic } \\
\text { basin }\end{array}$ & $\begin{array}{l}\text { Principal } \\
\text { land use }\end{array}$ & $\begin{array}{c}\text { Year } \\
\text { sampled }\end{array}$ & $\begin{array}{c}\text { Number } \\
\text { of sites } \\
\text { sampled }\end{array}$ \\
\hline Walker River & Walker & $\begin{array}{l}\text { Agriculture, } \\
\text { range }\end{array}$ & $\begin{array}{l}2012 \\
2019\end{array}$ & ${ }^{2} 4,6$ \\
\hline Truckee River & Truckee & Urban & 2012 & 2 \\
\hline $\begin{array}{l}\text { Stillwater } \\
\text { agricultural } \\
\text { ditches and } \\
\text { drains }\end{array}$ & Carson & 12 & 2014 & 6 \\
\hline Humboldt River & Humboldt & 9 & 2015 & 6 \\
\hline Virgin River ${ }^{1}$ & $\begin{array}{l}\text { Lower Colorado- } \\
\text { Lake Mead }\end{array}$ & 70 & 2017 & 2 \\
\hline Muddy River ${ }^{1}$ & $\begin{array}{l}\text { Lower Colorado- } \\
\text { Lake Mead }\end{array}$ & 235 & 2017 & 1 \\
\hline $\begin{array}{l}\text { Las Vegas } \\
\text { Wash }^{1}\end{array}$ & $\begin{array}{l}\text { Lower Colorado- } \\
\text { Lake Mead }\end{array}$ & 24 & 2017 & 1 \\
\hline Carson River & Carson & 25 & 2018 & 4 \\
\hline
\end{tabular}

${ }^{1}$ Pesticide sample collected discretely.

${ }^{2}$ Two sites sampled in 2012 were resampled in 2019.

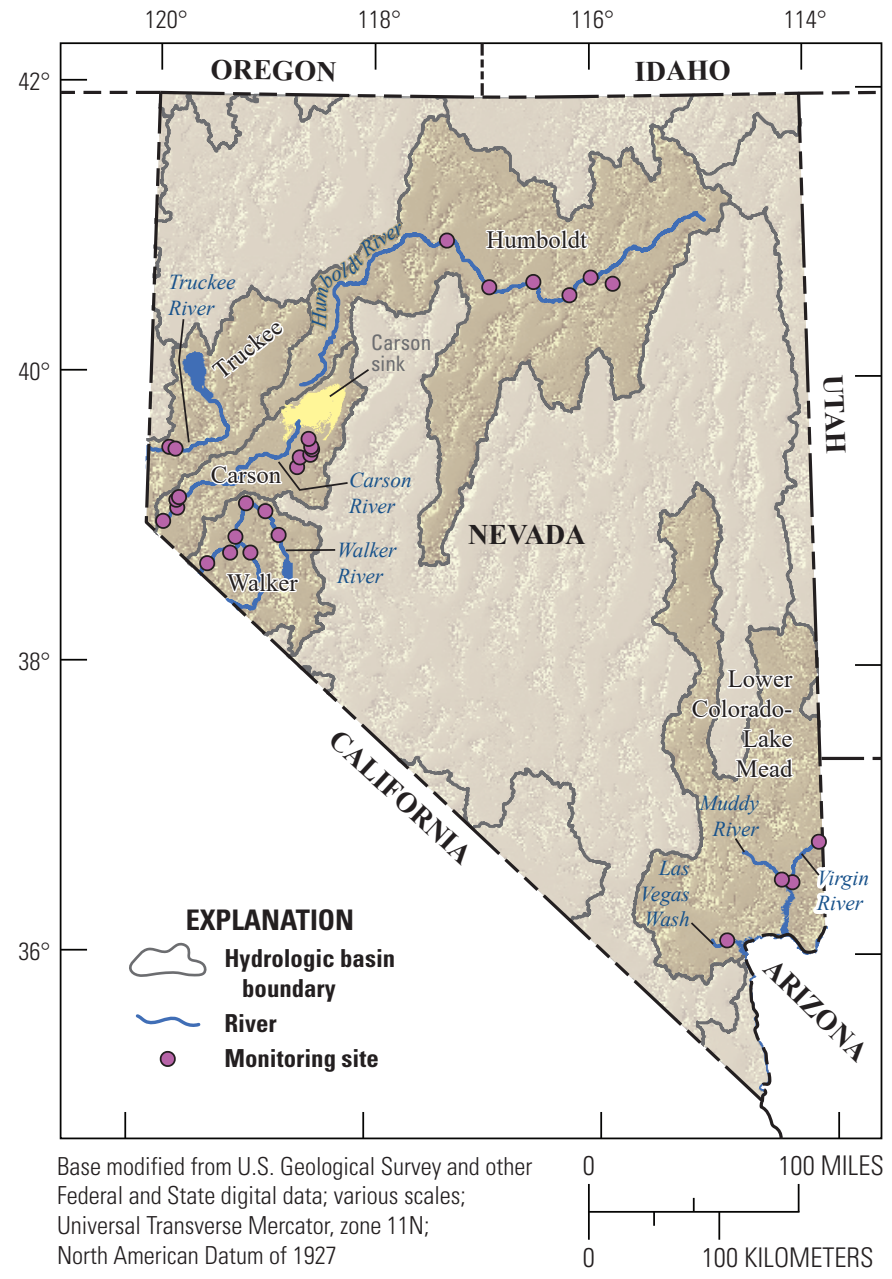

Figure 3. Pesticide monitoring areas in Nevada. Access site information and available data here. https://maps.waterdata.usgs. gov/mapper/nwisquery.html?URL=https://waterdata.usgs.

Glyphosate is a common and widely used herbicide for weed control both in residential areas and croplands. (U.S. Environmental Protection Agency, 2019). The EPA has set a maximum contamination level (MCL) of 700 micrograms per liter $(\mu \mathrm{g} / \mathrm{L})$ of glyphosate in drinking water. Kidney and reproductive damage have been documented as a couple of the possible health effects that could result from long-term exposure to drinking water with concentrations above the MCL (U.S. Environmental Protection Agency, 1995). In 2018, glyphosate was added to the monitoring effort to help the NDA understand glyphosate application practices by homeowners and farmers. Because POCIS samples can not be analyzed for glyphosate, discrete samples were collected from the Carson (2018) and Walker Rivers (2019) and analyzed for glyphosate at the USGS Pesticide Laboratory in Kansas.

\section{Results and Discussion}

\section{Pesticide Results from Passive Monitoring}

Each POCIS was analyzed for nearly 80 pesticides by the NDA chemistry laboratory in Sparks, Nevada. Results of selected passively collected pesticides are summarized in table 2. Analytical results received from the NDA laboratory were reported in nanograms/POCIS (ng/POCIS), however a conversion calculation (Alvarez, 2010) was performed to 
Table 2. Summary of Nevada Department of Agriculture's pesticide monitoring program analytical results from POCIS samples. All pesticides listed are on NDA's pesticides of concern list.

[herb, herbicide; insect, insecticide; $\mu \mathrm{g} / \mathrm{L}$, micrograms per liter; $\mathrm{U}$, analyzed for but not detected; NA, not available]

\begin{tabular}{|c|c|c|c|c|c|c|c|c|}
\hline $\begin{array}{c}\text { USGS station } \\
\text { identification } \\
\text { number }\end{array}$ & Station name & $\begin{array}{l}2,4-D \\
\text { (herb) }\end{array}$ & $\begin{array}{l}\text { Atrazine } \\
\text { (herb) }\end{array}$ & $\begin{array}{l}\text { Bromacil } \\
\text { (herb) }\end{array}$ & $\begin{array}{l}\text { Diuron } \\
\text { (herb) }\end{array}$ & $\begin{array}{l}\text { Hexazinone } \\
\text { (herb) }\end{array}$ & $\begin{array}{l}\text { Imidacloprid } \\
\text { (insect) }\end{array}$ & $\begin{array}{l}\text { Oxamyl } \\
\text { (insect) }\end{array}$ \\
\hline \multicolumn{9}{|c|}{$\mu \mathrm{g} / \mathrm{L}$} \\
\hline \multicolumn{2}{|c|}{ EPA Maximum contaminant level ${ }^{1}$} & 70 & 3 & $\mathrm{NA}^{2}$ & $\mathrm{NA}^{2}$ & $\mathrm{NA}^{2}$ & $\mathrm{NA}^{2}$ & 200 \\
\hline \multicolumn{2}{|c|}{ EPA Health advisory level ${ }^{1}$} & 200 & 700 & 3,500 & 100 & 2,000 & $\mathrm{NA}^{2}$ & 35 \\
\hline \multicolumn{2}{|c|}{ USGS Noncancer Health-based screening level ${ }^{3}$} & NA & NA & 100 & 20 & 300 & NA & NA \\
\hline \multicolumn{9}{|c|}{ Walker and Truckee River (2012) } \\
\hline 10297500 & $\begin{array}{l}\text { W Walker Rv blw Smith Vly Div nr } \\
\text { Wellington, NV }\end{array}$ & 0.0011 & 0.0003 & $\mathrm{U}$ & 0.0017 & $\mathrm{U}$ & $\mathrm{U}$ & $\mathrm{U}$ \\
\hline 10300000 & W Walker Rv nr Hudson, NV & 0.0033 & 0.0004 & $\mathrm{U}$ & 0.0019 & 0.0137 & $\mathrm{U}$ & $\mathrm{U}$ \\
\hline 10300600 & $\begin{array}{l}\text { Walker Rv at Snyder Ln nr Mason, } \\
\text { NV }\end{array}$ & 0.0035 & 0.0006 & $\mathrm{U}$ & 0.0122 & 0.0122 & $\mathrm{U}$ & $\mathrm{U}$ \\
\hline 10301500 & Walker Rv nr Wabuska, NV & 0.0030 & 0.0061 & $\mathrm{U}$ & 0.0068 & 0.0068 & 0.0052 & $\mathrm{U}$ \\
\hline 10348000 & Truckee Rv at Reno, NV & $\mathrm{U}$ & 0.0002 & 0.0034 & $\mathrm{U}$ & $\mathrm{U}$ & $\mathrm{U}$ & $\mathrm{U}$ \\
\hline 10348200 & Truckee Rv nr Sparks, NV & $\mathrm{U}$ & $\mathrm{U}$ & $\mathrm{U}$ & $\mathrm{U}$ & $\mathrm{U}$ & $\mathrm{U}$ & $\mathrm{U}$ \\
\hline \multicolumn{9}{|c|}{ Stillwater agricultural ditches and drains (2014) } \\
\hline 10312190 & $\begin{array}{l}\text { Lower Diagonal Drain at HWY } 50 \mathrm{nr} \\
\text { Fallon, NV }\end{array}$ & 0.0121 & $\mathrm{U}$ & 0.0627 & 0.0108 & $\mathrm{U}$ & $\mathrm{U}$ & $\mathrm{U}$ \\
\hline 1031220130 & $\begin{array}{l}\text { Harmon Reservoir Outflow nr Fal- } \\
\text { lon, NV }\end{array}$ & 0.0143 & $\mathrm{U}$ & 0.0107 & 0.0083 & 0.0005 & $\mathrm{U}$ & $\mathrm{U}$ \\
\hline 103122155 & $\begin{array}{l}\text { Stillwater Pt Res Bypass Canal nr } \\
\text { Stillwater, NV }\end{array}$ & 0.0143 & $\mathrm{U}$ & 0.0352 & 0.0118 & 0.0006 & $\mathrm{U}$ & $\mathrm{U}$ \\
\hline 1031221902 & $\begin{array}{l}\text { S-Line Diversion Canal near Stillwa- } \\
\text { ter, NV }\end{array}$ & 0.0187 & $\mathrm{U}$ & 0.0122 & 0.0069 & 0.0007 & $\mathrm{U}$ & $\mathrm{U}$ \\
\hline 10312220 & $\begin{array}{l}\text { Stillwater Slough Cutoff Drain nr } \\
\text { Stillwater, NV }\end{array}$ & 0.0231 & $\mathrm{U}$ & $\mathrm{U}$ & 0.0020 & 0.0008 & $\mathrm{U}$ & $\mathrm{U}$ \\
\hline 10312270 & $\begin{array}{l}\text { Paiute Drain at Wildlife Ent nr Still- } \\
\text { water, NV }\end{array}$ & 0.0593 & $\mathrm{U}$ & $\mathrm{U}$ & 0.0024 & $\mathrm{U}$ & $\mathrm{U}$ & $\mathrm{U}$ \\
\hline
\end{tabular}

\begin{tabular}{|c|c|c|c|c|c|c|c|c|}
\hline \multicolumn{9}{|c|}{ Humboldt River (2015) } \\
\hline 10320000 & $\begin{array}{l}\text { S Fk Humboldt Rv abv Dixie Ck nr } \\
\text { Elko, NV }\end{array}$ & $\mathrm{U}$ & $\mathrm{U}$ & $\mathrm{U}$ & $\mathrm{U}$ & $\mathrm{U}$ & $\mathrm{U}$ & $\mathrm{U}$ \\
\hline 10321000 & Humboldt Rv nr Carlin, NV & 0.0242 & $\mathrm{U}$ & $\mathrm{U}$ & 0.0007 & $\mathrm{U}$ & $\mathrm{U}$ & $\mathrm{U}$ \\
\hline 10322500 & Humboldt Rv at Palisade, NV & 0.0113 & $\mathrm{U}$ & $\mathrm{U}$ & 0.0113 & $\mathrm{U}$ & $\mathrm{U}$ & $\mathrm{U}$ \\
\hline 10323425 & $\begin{array}{l}\text { Humboldt Rv at Old US } 40 \text { Brg at } \\
\text { Dunphy, NV }\end{array}$ & 0.0344 & $\mathrm{U}$ & $\mathrm{U}$ & 0.0008 & $\mathrm{U}$ & $\mathrm{U}$ & $\mathrm{U}$ \\
\hline 10325000 & $\begin{array}{l}\text { Humboldt Rv at Battle Mountain, } \\
\text { NV }\end{array}$ & 0.0265 & $\mathrm{U}$ & $\mathrm{U}$ & 0.0006 & $\mathrm{U}$ & $\mathrm{U}$ & $\mathrm{U}$ \\
\hline 10327500 & Humboldt Rv at Comus, NV & 0.0053 & $\mathrm{U}$ & $\mathrm{U}$ & 0.0005 & $\mathrm{U}$ & $\mathrm{U}$ & $\mathrm{U}$ \\
\hline \multicolumn{9}{|c|}{ Carson River (2018) } \\
\hline 10310407 & Carson Rv nr Genoa, NV & 0.0151 & $\mathrm{U}$ & $\mathrm{U}$ & $\mathrm{U}$ & $\mathrm{U}$ & $\mathrm{U}$ & $\mathrm{U}$ \\
\hline 10311000 & Carson Rv nr Carson City, NV & 0.0056 & $\mathrm{U}$ & $\mathrm{U}$ & 0.0001 & 0.0003 & $\mathrm{U}$ & $\mathrm{U}$ \\
\hline 10311300 & Eagle Valley Ck at Carson City, NV & 0.0047 & 0.00005 & 0.0058 & 0.0139 & 0.0007 & 0.0001 & $\mathrm{U}$ \\
\hline 10311400 & $\begin{array}{l}\text { Carson Rv at Deer Run Rd nr Carson } \\
\text { City, NV }\end{array}$ & 0.0081 & $\mathrm{U}$ & $\mathrm{U}$ & 0.0004 & 0.0003 & 0.0006 & $\mathrm{U}$ \\
\hline
\end{tabular}


Table 2. Summary of Nevada Department of Agriculture's pesticide monitoring program analytical results from POCIS samples. All pesticides listed are on NDA's pesticides of concern list.-Continued

[herb, herbicide; insect, insecticide; $\mu \mathrm{g} / \mathrm{L}$, micrograms per liter; NV, Nevada; U, analyzed for but not detected; NA, not available]

\begin{tabular}{|c|c|c|c|c|c|c|c|c|}
\hline $\begin{array}{c}\text { USGS station } \\
\text { identification } \\
\text { number }\end{array}$ & Station name & $\begin{array}{l}\text { 2,4-D } \\
\text { (herb) }\end{array}$ & $\begin{array}{c}\text { Atrazine } \\
\text { (herb) }\end{array}$ & $\begin{array}{c}\text { Bromacil } \\
\text { (herb) }\end{array}$ & $\begin{array}{l}\text { Diuron } \\
\text { (herb) }\end{array}$ & $\begin{array}{c}\text { Hexazinone } \\
\text { (herb) }\end{array}$ & $\begin{array}{l}\text { Imidacloprid } \\
\text { (insect) }\end{array}$ & $\begin{array}{l}\text { Oxamyl } \\
\text { (insect) }\end{array}$ \\
\hline \multicolumn{9}{|c|}{ Walker River (2019) } \\
\hline 10293500 & $\begin{array}{l}\text { E Walker Rv abv Strosnider } \\
\text { Ditch nr Mason, NV }\end{array}$ & 0.0036 & $\mathrm{U}$ & $\mathrm{U}$ & $\mathrm{U}$ & $\mathrm{U}$ & $\mathrm{U}$ & $\mathrm{U}$ \\
\hline 10297500 & $\begin{array}{l}\text { W Walker Rv at Hoye Brg nr } \\
\text { Wellington, NV }\end{array}$ & 0.0003 & $\mathrm{U}$ & $\mathrm{U}$ & $\mathrm{U}$ & $\mathrm{U}$ & $\mathrm{U}$ & $\mathrm{U}$ \\
\hline 10300600 & $\begin{array}{l}\text { Walker Rv at Snyder Ln nr } \\
\text { Mason, } \mathrm{NV}^{4}\end{array}$ & 0.0021 & 0.0041 & $\mathrm{U}$ & $\mathrm{U}$ & 0.0001 & 0.0001 & 0.0001 \\
\hline 10301500 & Walker Rv nr Wabuska, NV ${ }^{4}$ & 0.0273 & 0.0052 & $\mathrm{U}$ & $\mathrm{U}$ & 0.0001 & 0.0106 & 0.0050 \\
\hline 10302002 & $\begin{array}{l}\text { Walker Rv at Lateral 2-A } \\
\text { Siphon nr Schurz, NV }\end{array}$ & 0.0044 & 0.0046 & $\mathrm{U}$ & $\mathrm{U}$ & 0.00005 & 0.0044 & 0.0001 \\
\hline \multirow[t]{4}{*}{10301600} & $\begin{array}{l}\text { Walker Rv abv Weber Res nr } \\
\text { Schurz, NV }\end{array}$ & 0.0107 & 0.0038 & $\mathrm{U}$ & $\mathrm{U}$ & 0.0001 & 0.0064 & 0.0009 \\
\hline & Number of detections $s^{5}$ : & 23 & 10 & 6 & 18 & 12 & 6 & 4 \\
\hline & Minimum concentration: & 0.0003 & 0.00005 & 0.0011 & 0.0001 & 0.00005 & 0.0001 & 0.0001 \\
\hline & Maximum concentration: & 0.0593 & 0.0052 & 0.0627 & 0.0139 & 0.0068 & 0.0106 & 0.0050 \\
\hline
\end{tabular}

${ }^{1}$ U.S. Environmental Protection Agency, 2018.

${ }^{2}$ Maximum contaminant level not yet developed by EPA.

${ }^{3}$ Norman and others, 2018.

${ }^{4}$ Sampled in 2012 and 2019.

${ }^{5}$ Out of 26 sites.

convert the pesticide results to concentrations for comparison to EPA maximum contaminant and health advisory levels (table 2). The USGS noncancer health-based screening levels are also provided for comparison (Norman and others, 2018).

Herbicides, used to control weeds, were the most detected form of pesticide in Nevada's surface waters. The three herbicides detected most often were the general use (can be applied without a license) herbicides 2,4-D (88 percent of the sites sampled), diuron (69 percent of the sites sampled), and hexazinone (46 percent of the sites sampled), which are applied by farmers, commercial pest control operators, and homeowners to kill unwanted weeds (table 2). 2,4-D is a widely used selective herbicide, meaning it only kills unwanted broadleaf plants while most grasses and crops remain unharmed (U.S. Environmental Protection Agency, 2005). Although 2,4-D was detected in every watershed sampled except the Truckee River, the concentrations were at least three orders of magnitude less than the EPA's MCL for the herbicide. Similar to 2,4-D, diuron was present in every watershed except the Truckee River; however, diuron was typically found in main-stem river channels downstream from agricultural areas (75 percent of the river sites sampled (9 of 12), excluding the six Stillwater agricultural ditches and drains). Diuron was detected in each of the four Walker River sites sampled in 2012 (range of $0.0017-0.0122 \mu \mathrm{g} / \mathrm{L}$ ); however, diuron was not present in the Walker River when resampled in 2019. All measured concentrations of diuron were at least four orders of magnitude below the EPA's health advisory level (table 2). The third most detected pesticide, hexazinone, was detected in all rivers sampled, except for the Truckee and Humboldt Rivers, at concentrations far below the EPA's health advisory level of 2,000 $\mu \mathrm{g} / \mathrm{L}$ (table 2). The remaining two herbicides, atrazine and bromacil, were detected less frequently than 2,4-D, diuron, and hexazinone. Atrazine, a restricted-use (requires an applicators license) herbicide, was detected in most Walker River sites; concentrations were at least two orders of magnitude below the established $3 \mu \mathrm{g} / \mathrm{L} \mathrm{MCL}$ (table 2). Bromacil, another general use herbicide, was detected in four of the six agricultural canals and drainage ditches near Fallon, Nevada, which included the highest pesticide concentration $(0.0627 \mu \mathrm{g} / \mathrm{L})$ obtained from POCIS samplers. The only pesticides detected in the Truckee River were atrazine and bromacil at concentrations at least four orders of magnitude less than EPA's health advisory levels.

Imidacloprid and oxamyl, two insecticides on NDA's pesticides of concern list, had comparatively fewer detections than herbicides during this study. Imidacloprid is a general use insecticide that was found in the main channels of the Walker and Carson Rivers, downstream from urban areas, at concentrations from 0.0001 to $0.0106 \mu \mathrm{g} / \mathrm{L}$ (table 2). In 2019, oxamyl (a restricted-use insecticide) was only detected in the four mainstem Walker River samples downstream from Mason, Nevada. Measured oxamyl concentrations were low, with the highest concentration four orders of magnitude less than the EPA's health advisory level of $35 \mu \mathrm{g} / \mathrm{L}$ (table 2). 
Table 3. Summary of Nevada Department of Agriculture's pesticide monitoring program from discrete samples analyzed by the USGS National Water Quality Laboratory. Glyphosate samples were collected from the Carson and Walker Rivers only in 2018 and 2019 , respectively.

[herb, herbicide; insect, insecticide; <, less than; $\mu \mathrm{g} / \mathrm{L}$, micrograms per liter; - , not sampled for; NV, Nevada; EPA, U.S. Environmental Protection Agency; USGS, U.S. Geological Survey; NA, not available]

\begin{tabular}{|c|c|c|c|c|c|c|}
\hline $\begin{array}{c}\text { USGS station } \\
\text { identification } \\
\text { number }\end{array}$ & Station name & $\begin{array}{l}\text { Acephate } \\
\text { (insect) }\end{array}$ & $\begin{array}{l}\text { Fipronil } \\
\text { (insect) }\end{array}$ & $\begin{array}{l}\text { Imidacloprid } \\
\text { (insect) }\end{array}$ & $\begin{array}{l}\text { Terbuthylazine } \\
\text { (herb) }\end{array}$ & $\begin{array}{l}\text { Glyphosate } \\
\text { (herb) }\end{array}$ \\
\hline \multicolumn{7}{|c|}{$\mu \mathrm{g} / \mathrm{L}$} \\
\hline \multicolumn{2}{|c|}{ EPA maximum contaminant level ${ }^{1}$} & $\mathrm{NA}^{3}$ & $\mathrm{NA}^{3}$ & $\mathrm{NA}^{3}$ & $\mathrm{NA}^{3}$ & 700 \\
\hline \multicolumn{2}{|c|}{ EPA health advisory level ${ }^{1}$} & $\mathrm{NA}^{3}$ & $\mathrm{NA}^{3}$ & $\mathrm{NA}^{3}$ & $\mathrm{NA}^{3}$ & 70,000 \\
\hline \multicolumn{2}{|c|}{ USGS health-based screening level ${ }^{2}$} & - & - & - & 2 & 40 \\
\hline 09415090 & Virgin Rv at Mesquite, NV & $<0.01$ & $<0.004$ & $<0.016$ & $<0.0036$ & - \\
\hline 09415250 & $\begin{array}{l}\text { Virgin Rv abv Lake Mead } \\
\text { Nr Overton, NV }\end{array}$ & $<0.01$ & $<0.004$ & $<0.016$ & $<0.0036$ & - \\
\hline 09419507 & $\begin{array}{l}\text { Muddy Rv at Lewis Av- } \\
\text { enue at Overton, NV }\end{array}$ & $<0.01$ & $<0.004$ & $<0.016$ & $<0.0036$ & - \\
\hline 09419800 & $\begin{array}{l}\text { LV Wash Blw Lake Las } \\
\text { Vegas Nr Boulder City, } \\
\text { NV }\end{array}$ & 0.0834 & 0.0045 & 0.0331 & 0.0118 & - \\
\hline 10310407 & Carson Rv nr Genoa, NV & - & - & - & - & $<0.02$ \\
\hline 10311000 & $\begin{array}{l}\text { Carson Rv nr Carson City, } \\
\text { NV }\end{array}$ & - & - & - & - & 0.02 \\
\hline 10311300 & $\begin{array}{l}\text { Eagle Valley Ck at Carson } \\
\text { City, NV }\end{array}$ & - & - & - & - & 2.9 \\
\hline 10311400 & $\begin{array}{l}\text { Carson Rv at Deer Run Rd } \\
\text { nr Carson City, NV }\end{array}$ & - & - & - & - & 0.03 \\
\hline 10293500 & $\begin{array}{l}\text { E Walker Rv abv Strosnid- } \\
\text { er Ditch nr Mason, NV }\end{array}$ & - & - & - & - & $<0.02$ \\
\hline 10297500 & $\begin{array}{l}\text { W Walker Rv at Hoye Brg } \\
\text { nr Wellington, NV }\end{array}$ & - & - & - & - & $<0.02$ \\
\hline 10300600 & $\begin{array}{l}\text { Walker Rv at Snyder Ln nr } \\
\text { Mason, NV }\end{array}$ & - & - & - & - & $<0.02$ \\
\hline 10301500 & $\begin{array}{l}\text { Walker Rv nr Wabuska, } \\
\text { NV }\end{array}$ & - & - & - & - & 0.1 \\
\hline 10302002 & $\begin{array}{l}\text { Walker Rv at Lateral 2-A } \\
\text { Siphon nr Schurz, NV }\end{array}$ & - & - & - & - & 0.02 \\
\hline 10301600 & $\begin{array}{l}\text { Walker Rv abv Weber Res } \\
\text { nr Schurz, NV }\end{array}$ & - & - & - & - & 0.05 \\
\hline
\end{tabular}

${ }^{1}$ U.S. Environmental Protection Agency, 2018.

${ }^{2}$ Norman and others, 2018.

${ }^{3}$ Maximum contaminant level not yet developed by the EPA.

The greatest number of pesticide detections in a single POCIS sample were found in Eagle Valley Creek, a tributary to the Carson River in Carson City receiving urban runoff. This site, sampled in 2018, had detections of six of the seven pesticides listed on NDA's pesticides of concern list: five herbicides and one insecticide (table 2). All pesticide concentrations were well below EPA MCLs and health advisories (table 2).

\section{Pesticide Results from Discrete Monitoring}

In 2017, discrete samples were collected from the Virgin River, Muddy River, and the Las Vegas Wash, a large urban return-flow channel discharging to Lake Mead. No pesticides were detected above laboratory reporting levels from the Virgin or Muddy Rivers; however, three insecticides and one herbicide were detected from the Las Vegas Wash: acephate, fipronil, imidacloprid, and terbuthylazine (table 3). None of these pesticides have established EPA MCLs or health advisories.

In 2018, discrete glyphosate samples were collected from the Carson River, and in 2019, from the Walker River. Glyphosate was detected at three sites on the Carson River and Walker River, each (ranging from 0.02 to $2.9 \mu \mathrm{g} / \mathrm{L}$ ) far below the $700 \mu \mathrm{g} / \mathrm{L}$ MCL (table 3). 


\section{Summary}

The Nevada Department of Agriculture (NDA), in cooperation with the U.S. Environmental Protection Agency (EPA), has created a pesticide management program for Nevada's groundwater and surface-water resources. During the past 8 years, the USGS has partnered with the NDA to monitor pesticides in groundwater and surface water as part of that program.

From 2012 to 2019, passive samplers have been used to monitor polar pesticides that possess chemical characteristics making them susceptible to partitioning into the State's water resources. To date, four Nevada rivers and various agricultural canals and drainage ditches near Fallon, Nevada, have been evaluated. Herbicides were the most frequently detected form of pesticide in Nevada's surface waters, including 2,4-D (23 of 26 sites), diuron (18 of 26 sites), and hexazinone (12 of 26 sites).The highest pesticide concentration detected using POCIS sampling technique was bromacil $(0.0627 \mu \mathrm{g} / \mathrm{L})$. This sample was collected from an agricultural drain near Fallon, Nevada, in 2014.

Using discrete sampling methods, three insecticides (acephate, fipronil, imidacloprid) and one herbicide (terbuthylazine) were found in Las Vegas Wash surface water. Generally, the concentrations of these pesticides $(0.0045$ to $0.0834 \mathrm{ug} / \mathrm{L})$ were within the same range as other pesticides $(0.00005$ to $0.0627 \mathrm{ug} / \mathrm{L}$ ) found in other Nevada surface waters using passive sampling. As of 2020, no established EPA drinking water criteria or health advisories have been established for these compounds. No pesticides were detected in either of the two tributaries to Lake Mead, the Virgin and Muddy rivers. In 2018 and 2019, samples from the Carson and Walker rivers, respectively, were analyzed for the very popular general-use herbicide glyphosate using discrete methods. Glyphosate was detected at three sites each on the Carson and Walker rivers; all concentrations were several orders of magnitude below the MCL. Pesticide concentrations typically are present at concentrations elusive to discrete sampling methods; therefore, monitoring of pesticides using discrete techniques is often ineffective at conclusively determining the presence or absence of pesticides. Overall, pesticides detected thus far in Nevada rivers have been at very low concentrations, orders of magnitude below established EPA MCLs and health advisories.

\section{Acknowledgments}

The authors extend sincere gratitude to Mr. Bret Allen from the Nevada Department of Agriculture for his leadership, fieldwork, and infectious good humor throughout the course of this project.

By Jena M. Huntington ${ }^{1}$, Derek C. Entz ${ }^{2}$, and Carl E. Thodal ${ }^{3}$

\footnotetext{
${ }^{1}$ U.S. Geological Survey, Nevada Water Science Center

${ }^{2}$ Nevada Department of Agriculture

${ }^{3}$ Retired, U.S. Geological Survey, Nevada Water Science Center
}

\section{References Cited}

Alvarez, D.A., 2010, Guidelines for the use of the semipermeable membrane device (SPMD) and the polar organic chemical integrative sampler (POCIS) in environmental monitoring studies: U.S. Geological Survey, Techniques and Methods 1-D4, 28 p., https://pubs. er.usgs.gov/publication/tm1D4

Atwood, D. and Paisley-Jones, Claire, 2017, Pesticides industry sales and usage 2008-2012 market estimates: U.S. Environmental Protection Agency report, accessed Dec. 11, 2020, at https://www.epa.gov/ pesticides/pesticides-industry-sales-and-usage-2008-2012-marketestimates.

Hefner, Melody, and Donaldson, Susan, 2006, What to do about fertilizers and pesticides: Reno, University of Nevada, Nonpoint Education for Municipal Officials, Protecting our Water Action Guide \#3: Fact Sheet-06-44, 2 p.

Keith, L., 1991, Environmental sampling and analysis-A practical guide: Boca Raton, Fl, CRC Press, 143 p.

Nevada Department of Agriculture, 2019, Groundwater Monitoring for Pesticides: Frequently Asked Questions, accessed July 31, 2020, at http://agri.nv.gov/uploadedFiles/agrinvgov/Content/Media/groundwater_monitoring_faq_r1.pdf.

Norman, J.E., Toccalino, P.L., Morman, S.A., 2018, Health-based screening levels for evaluating water-quality data ( $2 \mathrm{~d}$ ed.), U.S. Geological Survey Web page, https://water.usgs.gov/waterresources/hbsl/.

Thodal, C.E., Carpenter, J., and Moses, C.W., 2009, Monitoring for pesticides in groundwater and surface water in Nevada, 2008: U.S. Geological Survey Fact Sheet 2009-3093, 4p., https://pubs. er.usgs.gov/publication/fs20093093.

U.S. Environmental Protection Agency, 1995, National Primary Drinking Water Regulations, Glyphosate; U.S. Environmental Protection Agency EPA 811-F-95-003 a-T, accessed August 10, 2020 at https://nepis.epa.gov.

U.S. Environmental Protection Agency, 2005, 2,4-D RED facts: U.S. Environmental Protection Agency, EPA-738-F-05-002, 11 p., accessed December 10, 2020, at https://www3.epa.gov/pesticides/ chem_search/reg_actions/reregistration/fs_PC-030001_30-Jun-05.pdf

U.S. Environmental Protection Agency, 2018, 2018 edition of the drinking water standards and health advisories tables: U.S. Environmental Protection Agency, EPA 882-F-18-001, 20 p., accessed August 12, 2020, at https:/www.epa.gov/sdwa/2018-drinking-water-standardsand-advisory-tables

U.S. Environmental Protection Agency, 2019, Glyphosate memorandum, accessed Dec. 10, 2020, at https:/www.epa.gov/sites/production/files/2019-04/documents/glyphosate-response-comments-usagebenefits-final.pdf. 